\title{
A narrative review of intraoperative floppy iris syndrome: an update 2020
}

\author{
Xue Yang ${ }^{1}$, Zhaochuan Liu ${ }^{2}$, Zhigang Fan ${ }^{1}$, Andrzej Grzybowski ${ }^{3,4}$, Ningli Wang ${ }^{2}$ \\ ${ }^{1}$ State Key Laboratory of Ophthalmology, Zhongshan Ophthalmic Center, Sun Yat-sen University, Guangzhou, China; ${ }^{2}$ Beijing Institute of \\ Ophthalmology, Beijing Tongren Eye Center, Beijing Tongren Hospital, Capital Medical University, Beijing Ophthalmology and Visual Science \\ Key Lab, Beijing, China; ${ }^{3}$ Chair of Ophthalmology, University of Warmia and Mazury, Olsztyn, Poland; Institute for Research in Ophthalmology, \\ Poznan, Poland \\ Contributions: (I) Conception and design: A Grzybowski, N Wang; (II) Administrative support: N Wang; (III) Provision of study materials or patients: \\ None; (IV) Collection and assembly of data: X Yang, Z Liu; (V) Data analysis and interpretation: X Yang, Z Fan, A Grzybowski; (VI) Manuscript \\ writing: All authors; (VII) Final approval of manuscript: All authors. \\ Correspondence to: Prof. Ningli Wang. Beijing Institute of Ophthalmology, Beijing Tongren Eye Center, Beijing Tongren Hospital, Capital Medical \\ University; Beijing Ophthalmology and Visual Science Key Lab, Beijing, China, No. 1 Dongiiaominxiang Street, Dongcheng District, Beijing, \\ China. Email: wningli@vip.163.com.
}

\begin{abstract}
Intraoperative floppy iris syndrome (IFIS) is characterized by intraoperative floppiness or billowing of the iris, progressive miosis, and iris prolapse through the surgical wounds. It was originally reported about fifteen years ago, which was later identified to be closely associated with tamsulosin, the most commonly used $\alpha 1$ adrenoceptor antagonist for benign prostatic hyperplasia (BPH). A variety of risk factors, including age, gender, hypertension, axial length of the eye, $5 \alpha$-reductase inhibitors, other $\alpha$ adrenoceptor antagonist and neuromodulators, have been connected with IFIS. If IFIS occurs during phacoemulsification surgery, complications such as corneal endothelial loss, iris trauma, posterior capsule rupture (PCR), high intraocular pressure and vitreous loss are significantly increased. Therefore, preoperative evaluation of high-risk patients and appropriate intraoperative intervention is crucial to avoid severe complications. This review summarizes the pathogenesis and clinical features of classic IFIS, and provides some clinical pearls to ophthalmologists that may help identify, prevent or reduce IFIS associated complications. Additionally, from the perspective of clinical occurrence of IFIS, there are some recommendations for urologists as well. In conclusion, both ophthalmologists and urologists should be aware of this special clinical situation and communicate with each other about their own fields. A multidisciplinary interaction is of importance to simplify potentially complicated clinical issues.
\end{abstract}

Keywords: Intraoperative floppy iris syndrome (IFIS); cataract surgery; $\alpha 1$ adrenoceptor antagonist; risk factors

Submitted Apr 08, 2020. Accepted for publication Aug 07, 2020.

doi: $10.21037 /$ atm-20-3214

View this article at: http://dx.doi.org/10.21037/atm-20-3214

\section{Introduction}

Intraoperative floppy iris syndrome (IFIS), first described by Chang and Campbell, is defined as a triad of intraoperative signs: billowing of a floppy iris stroma under normal fluidics in the anterior chamber, propensity for iris prolapse through surgical incisions, and progressive intraoperative miosis despite adequate use of mydriatics (1) (Figure 1). IFIS primarily occurs during cataract surgery. Based on the signs observed intraoperatively, IFIS is classified into four grades: none (no signs), mild (billowing only), moderate (billowing plus either iris prolapse or progressive miosis) and severe (all the signs) (2).

Numerous studies have connected tamsulosin, a selective $\alpha 1$ adrenoceptor antagonist, with the occurrence of IFIS (1,3-6). Other potential risk factors include age, gender, race, axial length of the eye, ocular comorbidities such as 
A
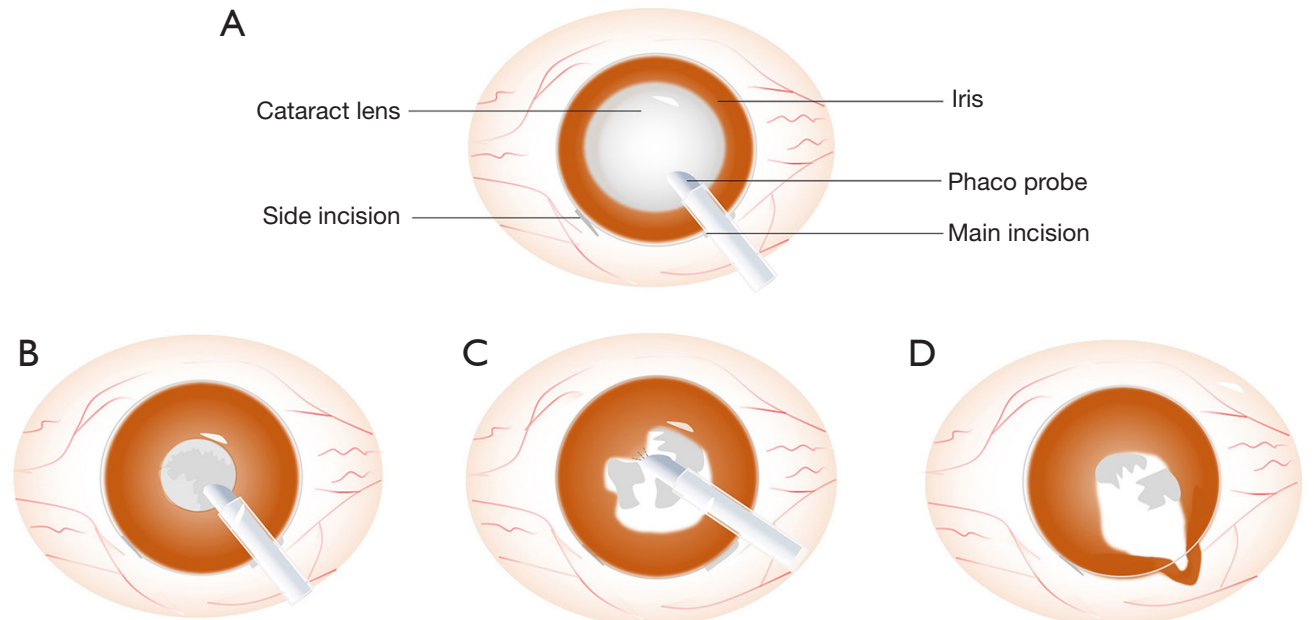

Figure 1 Three characteristics of IFIS. (A) Stable status of mydriasis during a normal cataract surgery. (B,C) Three characteristics of IFIS. (B) Progressive intraoperative miosis; (C) billowing of a floppy iris stroma; (D) propensity for iris prolapse through surgical incisions. IFIS, intraoperative floppy iris syndrome.

pseudoexfoliation syndrome and glaucoma, hypertension, diabetes mellitus, $5 \alpha$-reductase inhibitors and other $\alpha$ adrenoceptor antagonist such as silodosin, alfuzosin, doxazosin, and several neuromodulators (2,7-12).

The occurrence of unanticipated IFIS is accompanied by increased rates of multiple complications, including corneal endothelial loss, iris trauma, anterior capsule tears, posterior capsule rupture (PCR), retained nuclear fragments, vitreous loss, macular edema and postoperative ocular inflammation $(1,13)$. These features make IFIS an important situation for ophthalmologists to prevent, recognize and manage appropriately. We present the following article in accordance with the NARRATIVE REVIEW reporting checklist (available at http://dx.doi.org/10.21037/atm-20-3214).

\section{Methodology}

Pertinent publications were identified through a systematic search of PubMed with the following key words: "intraoperative floppy iris syndrome", "cataract surgery", "phacoemulsification", " $\alpha 1$ adrenoceptor antagonist", and "tamsulosin". All related review articles, case reports and clinical studies were screened with no restriction on language and publication date. Data were extracted from each eligible article by 2 investigators working independently to analyze the ratio of IFIS in patients with a blockers including tamsulosin, terazosin, doxazosin and alfuzosin.

\section{Discussion}

\section{Epidemiology}

The prevalence of IFIS among patients undergoing cataract surgery varies by populations and races. Previous data shows that the overall incidence of IFIS ranges from $2 \%$ to $3 \%$ in the United States (14) while this number is up to $12.6 \%$ in Austria (15). Prospective observational studies show the incidence of IFIS is only $0.8 \%$ in Korea (16) and 3.18\% in China (17). Most IFIS cases reported occur in patients with a medical history of using $\alpha 1$ adrenoceptor antagonist, especially systemic tamsulosin. Tamsulosin is regarded as the first-line medical treatment for benign prostatic hyperplasia (BPH). Existing data show the incidence of $\mathrm{BPH}$ is approximately $50 \%$ in men over age 50 years, while this number increases to more than $80 \%$ in men over their eighth decade of life (18). Cataract also increases with aging. The prevalence of cataract is $3.9 \%$ at age $55-64$ years old while this number increases up to $92.6 \%$ at age over 80 (19). Surgical removal of a cataract is a standard procedure of choice. In industrialized countries, the connection between IFIS and tamsulosin has been largely recognized due to repeated medical educations among both ophthalmologists and urologists, which partially contribute to reducing the incidence of IFIS associated surgical complications following specific preoperative and intraoperative interventions $(20,21)$. However, there still exists a knowledge gap about IFIS among urologists based on an assessment of American urologists' 


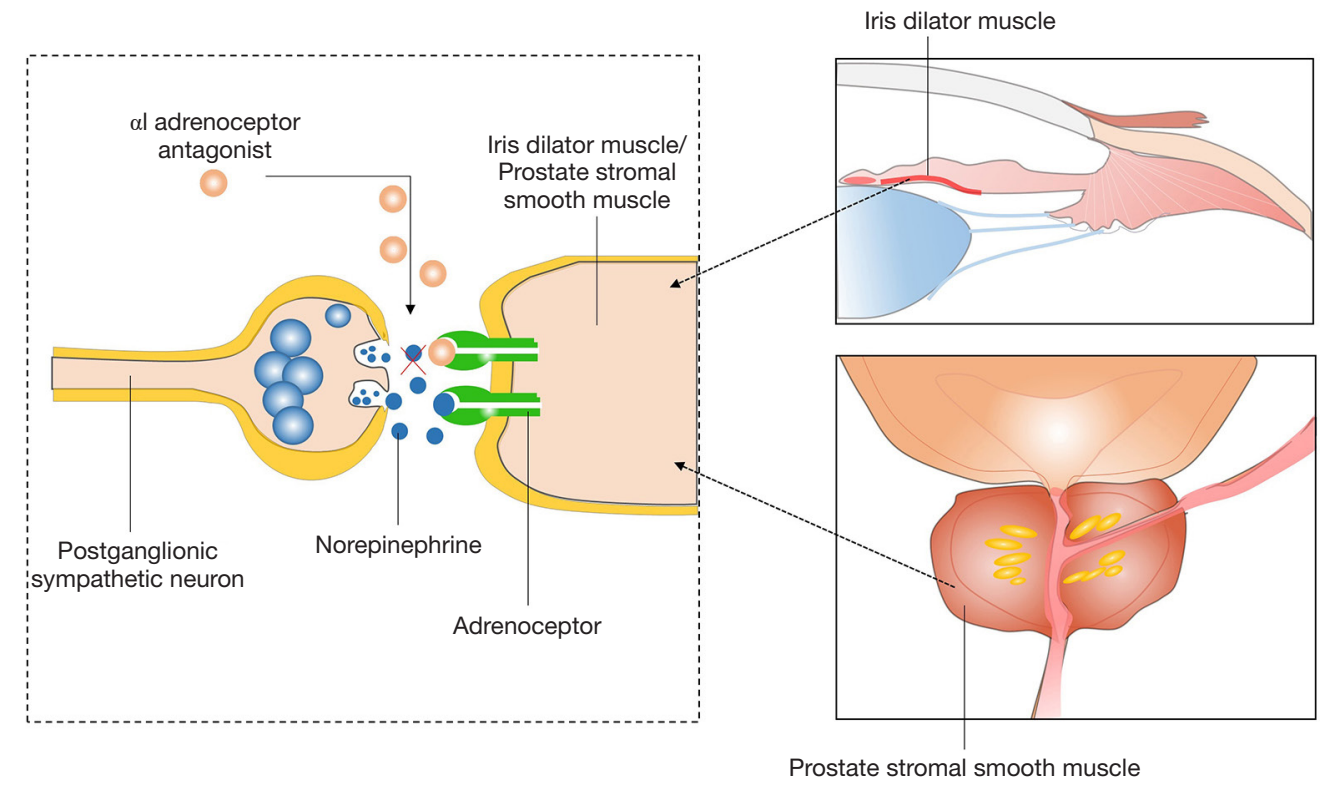

Figure 2 The mechanisms of $\alpha 1$ adrenoceptor antagonist on prostate and iris. $\alpha 1$ adrenoceptor antagonist competitively binds to $\alpha 1 \mathrm{~A}$ receptors on prostate stromal and iris dilator muscle resulting relaxation of urinary tract and iris miosis.

knowledge of IFIS (22), indicating that continuing education is required.

\section{Risk factors and pathogenesis}

\section{Medication}

Tamsulosin, a selective $\alpha 1$ adrenoceptor antagonist, was found to be strongly associated with IFIS. It has been reported that $57-100 \%$ of patients taking tamsulosin show at least one classic sign of IFIS during cataract surgery (23). In addition, a meta-analysis study reached the conclusion that odds ratio for IFIS after tamsulosin use was about 40fold higher than that after alfuzosin use (24).

There are three subtypes of $\alpha 1$ adrenoceptor including $\alpha 1 \mathrm{~A}, \alpha 1 \mathrm{~B}$ and $\alpha 1 \mathrm{D}$. Tamsulosin mainly blocks $\alpha 1 \mathrm{~A}$ adrenoceptor, which predominantly exist in prostate stromal smooth muscle, resulting in smooth muscle relaxation and subsequent relief of urinary tract symptoms (25). This particular receptor is present in iris dilator muscle as well (Figure 2). While oral tamsulosin improves the passage of urine, it results in miosis, loss of iris tone and appearance of IFIS. Clinically, other $\alpha 1$ adrenoceptor antagonist such as doxazosin, alfuzosin and terazosin also contribute to the occurrence of IFIS (1,26-29) (Table 1). However, the overall effect of these medicines in comparison with tamsulosin is less significant due to their difference in receptor affinity. Besides additional technical difficulty in phaco surgery, exposure to tamsulosin within 14 days of cataract surgery significantly increases serious postoperative complications, including loss of entire lens or lens fragment, retinal detachment or endophthalmitis (11). There are specific recommendations reached through survey and consensus among urologists. In short, urologists are asked to consider referring every patient with $\mathrm{BPH}$ for an assessment on potential cataract surgery before starting medical treatment (30).

Tamsulosin has a long half-life and its blocking effect to $\alpha 1$ adrenoceptor is irreversible. There are reports and clinical observations that IFIS can happen during cataract surgery even after tamsulosin therapy has been terminated years ago, which indicates that detrimental effect of tamsulosin to iris is likely permanent. An experimental study on 20 male Wistar rats shows that iris dilator muscle layer become thinner as the duration of treatment with tamsulosin increases (31). In clinical research, a crosssectional study reveals that iris thickness at the region of dilator muscle is significantly reduced in patients receiving tamsulosin, compared to that in control group (32). This finding has been corroborated by another retrospective study (33) and a prospective study (34). Damage and atrophy to iris dilator muscle, however, may not be exclusively due to $\alpha 1 \mathrm{~A}$ receptor blockade. Drug-melanin interaction and consequent immune response may be underlying 
Table 1 The rate of IFIS in studies

\begin{tabular}{|c|c|c|c|c|c|c|c|}
\hline Data source & Country & IFIS cases/all cases & IFIS cases/ $\alpha 1$ blocker group & Tamsulosin & Terazosin & Doxazosin & Alfuzosin \\
\hline Chadha (2007), (26) & UK & $29 / 1,786(1.6 \%)$ & $18.1 \%$ & $12 / 21$ & $0 / 1$ & $1 / 48$ & $0 / 2$ \\
\hline Srinivasan (2007), (27) & Canada & Unknown/1,298 & $20.0 \%$ & $10 / 18$ & $2 / 32$ & $1 / 17$ & $0 / 1$ \\
\hline Chang (2014), (2) & France & $100 / 226(44.2 \%)$ & $63.7 \%$ & $42 / 70$ & - & - & $30 / 43$ \\
\hline Chatziralli (2016), (9) & Greece & $63 / 1,274(4.9 \%)$ & $12.3 \%$ & $18 / 117$ & $6 / 55$ & - & $12 / 121$ \\
\hline Wahl (2017), (15) & Austria & 119/947 (12.6\%) & $83.3 \%$ & $11 / 11$ & $4 / 7$ & - & - \\
\hline Wen (2018), (29) & China & $53 / 482(11.0 \%)$ & $24.5 \%$ & $27 / 101$ & $9 / 34$ & $14 / 70$ & $8 / 32$ \\
\hline Kaczmarek (2019), (28) & Poland & $29 / 319(0.9 \%)$ & $28.6 \%$ & $8 / 18$ & - & $4 / 24$ & - \\
\hline
\end{tabular}

IFIS, intraoperative floppy iris syndrome.

Table 2 The medications related to IFIS

Drugs
$\alpha 1$ adrenoceptor antagonist
Tamsulosin
Doxazosin
Silodosin
Alfuzosin
Terazosin
$5 \alpha$-reductase inhibitors
Finasteride
Angiotensin II blockers
Neuromodulators
Benzodiazepine
Donepezil
Duloxetine
Antipsychotics
Quetiapine
Chlorpromazine
Zuclopenthixol
Ariprazole

IFIS, intraoperative floppy iris syndrome.

mechanisms as well (35). Iris vascular dysfunction may also be related to tamsulosin treatment, since $\alpha 1 \mathrm{~A}$ adrenoceptor are present within iris arteriolar muscularis too. Investigation into the ultrastructure pathology of iris from a tamsulosin-induced IFIS patient reveals pathological changes within iris arteriolar (36). The dysfunction in iris vasculature may increase the incidence of IFIS through disturbed blood supply or structure support (14). Finasteride as $5 \alpha$-reductase inhibitors is another type of medicine prescribed for $\mathrm{BPH}$, which has been suspected to cause IFIS (28). Therefore, ophthalmologists should pay attention to patients receiving finasteride too.

Angiotensin II receptor inhibitors are commonly prescribed for patients with cardiovascular diseases. Recently, a large comparative retrospective case-control study reveals that angiotensin II receptor inhibitors significantly contribute to the IFIS in women, which might be an independent risk factor for the development of IFIS (37). This result demonstrates IFIS is not a disease exclusive for men.

Other medications that have been reported to have increased risk for IFIS include neuromodulators such as onepezil, duloxetine, diazepines as well as several antipsychotics such as chlorpromazine, quetiapine, risperidone, zuclopenthixol and aripiprazole (9,28,38-40) (Table 2). The mechanism of these drugs causing IFIS is still unknown.

\section{Age and gender}

Although IFIS was reported during congenital cataract surgery in an infant (41), the elderly is the primary patients group. Aging has a positive association with the incidence of IFIS (28), consistent with increased prevalence of cataract and other aging related degenerative diseases. Gender is 


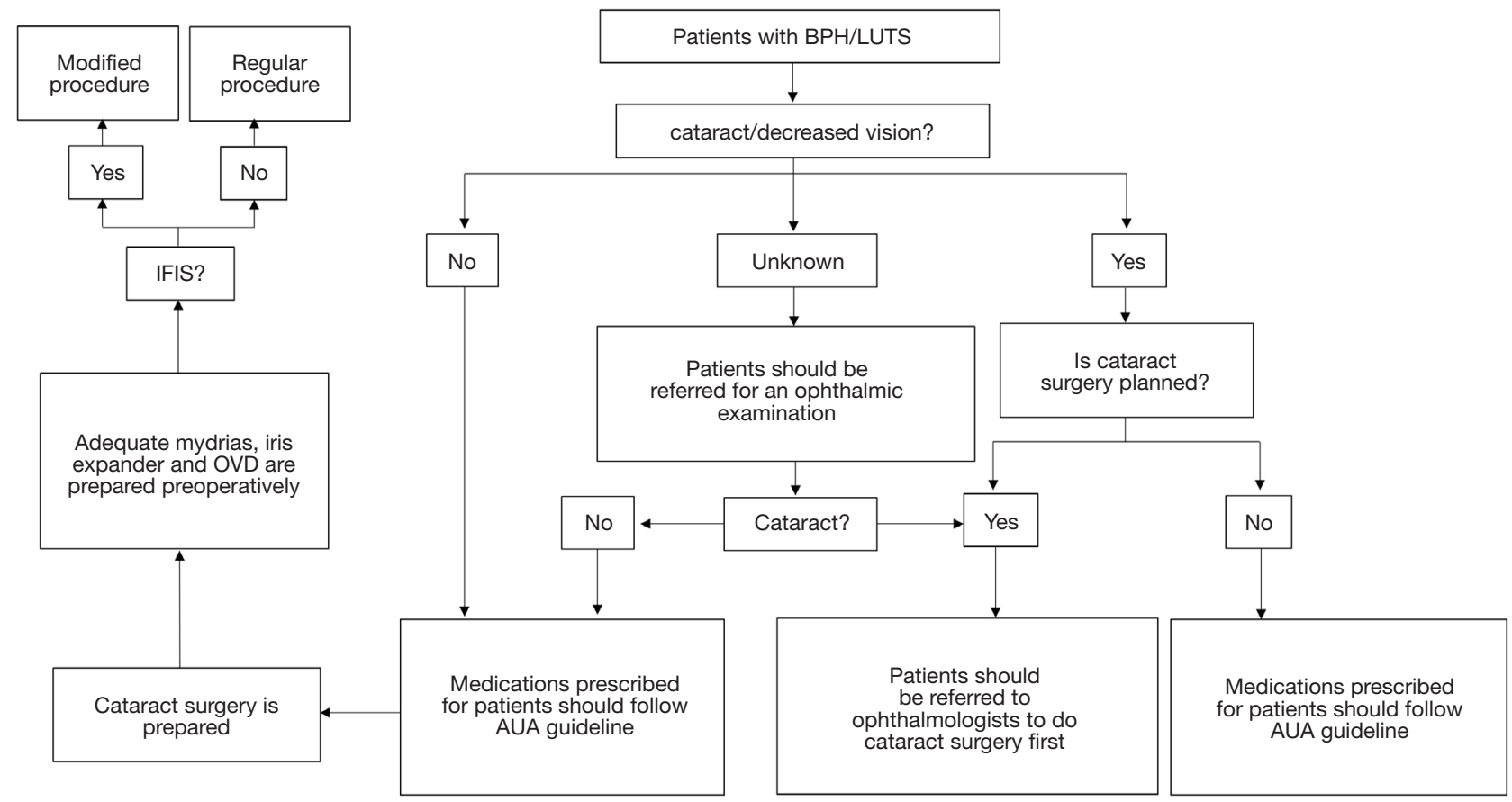

Figure 3 Flow chart recommended for urologists and ophthalmologists. BPH, benign prostatic hyperplasia; LUTS, lower urinary tract symptoms; IFIS, intraoperative floppy iris syndrome; OVD, ocular viscoelastic device; AUA, American Urological Association.

another risk factor. Many studies reveal that incidence of IFIS is significantly higher in male than in female $(10,28)$. The use of $\alpha 1$ adrenoceptor antagonist for men with BPH definitely increases the rate of IFIS. However, women also take this drug to treat lower urinary tract symptoms. It should be noted that the incidence of PCR was found significantly higher in female IFIS cases compared with male IFIS cases (10). Therefore, eye surgeons should recognize different predisposing factors among both male and female patients.

\section{Systemic diseases and other ocular conditions}

Hypertension and diabetes mellitus are two common diseases in the elderly. Diabetes mellitus has been reported to be unrelated to the occurrence of IFIS, while there are inconsistent results regarding the association of hypertension and IFIS. A study including 319 patients receiving phaco cataract surgery reveals no statistical connection between hypertension and IFIS (28). However, another prospective study consisting of 1,274 cataract patients reveals hypertension as an independent risk factor for IFIS (9).

Short axial length of the eye is significantly related to IFIS through a univariate analysis (9), indicating that anatomical features of ocular structure may be a potential risk factor for IFIS. Interestingly, Wahl et al. hypothesized that longer axial length might be a cofactor for IFIS since there is a relatively wider space for iris stretching and different flow dynamics forcing iris movement. However, they did not find any evidence supporting their hypothesis during their investigation (15). Shallow anterior chamber and convex iris configuration, anatomical features related to glaucoma, are predisposing factors for intraoperative iris prolapse during cataract surgery (42). However, there is no evidence that ocular comorbidities, including pseudoexfoliation syndrome and glaucoma etc., are associated with IFIS (28). Taken together, basic research and large-scale clinical studies are needed to explore the relationship between ocular conditions and IFIS.

\section{The management of IFIS}

\section{Preoperative evaluation}

Preoperative evaluation is crucial in preventing complications associated with IFIS. Stratification and assessment of risk factors for each patient is required to determine a personalized surgical strategy (Figure 3). A comprehensive medical history should be obtained and 
documented routinely before cataract surgery, especially specific medication prescription (tamsulosin and other $\alpha$ adrenoceptor antagonist). Once a risk factor of IFIS is confirmed, specific surgical modifications may be proactively prepared.

Preoperative use of topical atropine and epinephrine is beneficial for reducing IFIS. Epinephrine is more effective making it a better option for patients without cardiovascular diseases $(43,44)$. Preoperative pupil dilation should be reinforced with topical nonsteroidal Anti-inflammatory Drugs (NSAIDs), which can block cyclo-oxygenase and restrain miosis caused by prostaglandins release.

\section{Intraoperative measures}

Adequate pupil dilation is critical for phaco surgery in IFIS. Once preoperative prophylaxis is ineffective in high-risk patients or in an unanticipated occasion when IFIS occurs, modified surgical techniques are needed. The mixture of $1.0 \%$ phenylephrine and $0.3 \%$ ketorolac adding irrigation solution during surgery can prevent or reduce the severity of IFIS. However, this solution is created based on experience with Caucasian population, which may not be readily usable in other races (45). Intracameral phenylephrine can reduce iris floppiness and promote iris dilation, which can be recommended in most cases with progressive miosis. However, if iris dilator muscle is severely damaged, phenylephrine may not work at all. Micro-incision cataract surgery with incision less than $2 \mathrm{~mm}$ has many advantages, including stabilization of the anterior chamber, less tendency for iris prolapse and less intraoperative tissue injury with reduced intraocular inflammation. Small incision surgery can be useful for mild cases of IFIS, but its efficiency reduces in more severe cases (46). Some surgeons choose to use modified, longer-tunnel corneal incisions for phacoemulsification cataract surgery in highrisk patients $(47,48)$. Ocular viscoelastic devices (OVDs) are introduced to improve $\mathrm{Phaco}$, which are especially useful for intraoperative miosis after capsulorrhexis. OVD covers intraocular structure providing satisfactory iris stability and reserving space for pupillary devices if needed.

Other surgical pearls are helpful too. A moderate use of viscoelastic and special attention should be paid to avoid viscoelastic reaching over the pupillary margin, and seeping into posterior chamber. This is quite common in an IFIS patient due to iris and pupil floppiness. If viscoelastic accumulates within posterior chamber, it pushes iris forward, leading to repeated iris prolapse. When iris prolapse happens in an IFIS case, the surgeon should refrain from the natural impulse of inputting more viscoelastic inside anterior chamber to push down the iris. Instead, careful manipulation should be performed to release viscoelastic out of the posterior chamber. More effectively, if IFIS is highly suspected to happen through preoperative assessment, a pupil dilating device should be used preemptively, which commonly includes various types of iris retractors and pupil expanders. An iris retractor placed 1.5-2 mm behind and below the main phaco incision is the best and easiest option in preventing iris prolapse in IFIS. For a pupil expander, it is preferred to be inserted through clear corneal incision (2.2-2.8 mm), after OVD injection but before other surgical steps. Another thing to keep in mind in handling IFIS is that phaco machine should be set at a more "slow motion" dynamics. Floppy iris is more stable at a low IOP, low flow, and low vacuum setting (49). Attention should be constantly paid to keep the phaco tip away from iris and pupil margin, especially in cases with severe IFIS that have very small pupil under maximal madriatics (50).

\section{Recommendations for urologists}

A prospective trial has demonstrated that discontinuation of tamsulosin before cataract surgery can neither prevent the occurrence of IFIS nor improve the severity of IFIS, since the side effect of tamsulosin to iris is probably permanent (51). As aging is common worldwide, more people suffer from cataract as well as BPH/LUTS (lower urinary tract symptoms). Therefore, there are recommendations for urologists to avoid the use of tamsulosin when their patients are likely to receive cataract surgery in the near future (Figure 3). An assessment should be performed prior to the start of medical treatment to $\mathrm{BPH}$ to avoid the incidence of IFIS. Briefly, urologists should firstly ask their patients if they have been diagnosed with cataracts and if a surgery is planned for the near future (52). Secondly, for patients with decreased vision without previous ophthalmic evaluation, reference to an ophthalmologist should be recommended prior to starting long-term treatment with $\alpha 1$-blockers, especially tamsulosin (5). Thirdly, urologists are preferred to choose other medications such as finasteride rather than tamsulosin to treat patients with cataracts and BPH/LUTS (53). Fourthly, patients who are prescribed tamsulosin should be instructed to inform their ophthalmologists that they are taking the medicine. Most importantly, regular cross-talk must be built between urologists and ophthalmologists.

When patients with BPH/LUTS and mild to moderate 
cataract do not have plan to do cataract surgery soon or patients do not suffer from cataract-related vision decrease, urologists should follow American Urological Association (AUA) guideline to prescribe medicines for their patients. Patients with mild LUTS secondary to BPH are recommended for $\alpha$-blocker monotherapy while patients with symptomatic prostatic enlargement but without signs of bother may be offered a $5 \alpha$-reductase inhibitor. The combination of an $\alpha$ blocker and a $5 \alpha$-reductase inhibitor is an appropriate and effective treatment for patients with LUTS associated with manifest prostatic enlargement (54).

\section{Conclusions}

With improvement in medical and social conditions, aging is now worldwide, which is associated with an increased incidence of BPH and cataract. Since IFIS has a strong association with patients taking tamsulosin, a preferred treatment to BPH, it is of paramount importance for both ophthalmologists and urologists to raise awareness of their connection. Additionally, appropriate preoperative assessment of potential risks for IFIS and corresponding intraoperative measures are crucial to reduce complications.

\section{Acknowledgments}

Funding: None.

\section{Footnote}

Provenance and Peer Review: This article was commissioned by the Guest Editor (Dr. Andrzej Grzybowski) for the series "Recent developments in cataract surgery" published in Annals of Translational Medicine. The article was sent for external peer review organized by the Guest Editor and the editorial office.

Reporting Checklist: The authors have completed the Narrative Review reporting checklist. Available at http:// dx.doi.org/10.21037/atm-20-3214

Conflicts of Interest: All authors have completed the ICMJE uniform disclosure form (available at http:// dx.doi.org/10.21037/atm-20-3214). The series "Recent developments in cataract surgery" was commissioned by the editorial office without any funding or sponsorship. AG served as the unpaid Guest Editor of the series. The authors have no other conflicts of interest to declare.
Ethical Statement: The authors are accountable for all aspects of the work in ensuring that questions related to the accuracy or integrity of any part of the work are appropriately investigated and resolved.

Open Access Statement: This is an Open Access article distributed in accordance with the Creative Commons Attribution-NonCommercial-NoDerivs 4.0 International License (CC BY-NC-ND 4.0), which permits the noncommercial replication and distribution of the article with the strict proviso that no changes or edits are made and the original work is properly cited (including links to both the formal publication through the relevant DOI and the license). See: https://creativecommons.org/licenses/by-nc-nd/4.0/.

\section{References}

1. Chang DF, Campbell JR. Intraoperative floppy iris syndrome associated with tamsulosin. J Cataract Refract Surg 2005;31:664-73.

2. Chang DF, Campbell JR, Colin J, et al. Prospective masked comparison of intraoperative floppy iris syndrome severity with tamsulosin versus alfuzosin. Ophthalmology 2014;121:829-34.

3. Tiwari A. Tamsulosin and floppy iris syndrome in benign prostatic hyperplasia patients. Expert Opin Investig Drugs 2006;15:443-6.

4. Brogden PR, Backhouse OC, Saldana M. Intraoperative floppy iris syndrome associated with tamsulosin. Can Fam Physician 2007;53:1148.

5. Chang DF, Braga-Mele R, Mamalis N, et al. Clinical experience with intraoperative floppy-iris syndrome. Results of the 2008 ASCRS member survey. J Cataract Refract Surg 2008;34:1201-9.

6. Lunacek A, Mohamad Al-Ali B, Radmayr C, et al. Ten years of intraoperative floppy iris syndrome in the era of alpha-blockers. Cent European J Urol 2018;71:98-104.

7. Ipekci T, Akin Y, Hoscan B, et al. Intraoperative floppy iris syndrome associated with silodosin. Acta Ophthalmol 2015;93:e306.

8. Chatterjee S, Agrawal D. Silodosin-associated intraoperative floppy iris syndrome. Indian J Ophthalmol 2017;65:538-9.

9. Chatziralli IP, Peponis V, Parikakis E, et al. Risk factors for intraoperative floppy iris syndrome: a prospective study. Eye (Lond) 2016;30:1039-44.

10. Tzamalis A, Matsou A, Dermenoudi M, et al. The role of sex in intraoperative floppy-iris syndrome. J Cataract 
Refract Surg 2019;45:41-7.

11. Christou CD, Tsinopoulos I, Ziakas N, et al. Intraoperative Floppy Iris Syndrome: Updated Perspectives. Clin Ophthalmol 2020;14:463-71.

12. Dogan M, Kutluksaman B, Keles I, et al. The Effects of Systemic Alfuzosin and Tamsulosin Hydrochloride on Choroidal Thickness and Pupil Diameter Sizes in Cases with Benign Prostatic Hyperplasia. Curr Eye Res 2017;42:1638-43.

13. Storr-Paulsen A, Jorgensen JS, Norregaard JC, et al. Corneal endothelial cell changes after cataract surgery in patients on systemic sympathetic alpha-1a antagonist medication (tamsulosin). Acta Ophthalmol 2014;92:359-63.

14. Friedman AH. Tamsulosin and the intraoperative floppy iris syndrome. Jama 2009;301:2044-5.

15. Wahl M, Tipotsch-Maca SM, Vecsei-Marlovits PV. Intraoperative floppy iris syndrome and its association with various concurrent medications, bulbus length, patient age and gender. Graefes Arch Clin Exp Ophthalmol 2017;255:113-8.

16. Lim DH, Lee MG, Chung TY, et al. Korean patients taking $\alpha 1$-adrenergic receptor antagonists show lower incidence of intraoperative floppy iris syndrome than western patients. Br J Ophthalmol 2014;98:479-83.

17. Yu J, Yao K. Comparison of the effectiveness of two different pharmacologic approaches to prevent intraoperative floppy iris syndrome. International Journal of Ophthalmology 2017;17:2126-9.

18. Chughtai B, Forde JC, Thomas DD, et al. Benign prostatic hyperplasia. Nat Rev Dis Primers 2016;2:16031.

19. Liu YC, Wilkins M, Kim T, et al. Cataracts. Lancet 2017;390:600-12.

20. González Martín-Moro J, Santos Arrontes D, Martinez Silva V, et al. What urologists know about intraoperative floppy-iris syndrome. J Cataract Refract Surg 2010;36:2006-7.

21. Handzel DM, Briesen S, Rausch S, et al. Cataract surgery in patients taking alpha-1 antagonists: know the risks, avoid the complications. Dtsch Arztebl Int 2012;109:379-84.

22. Zhang Y, Shamie N, Daneshmand S. Assessment of Urologists' Knowledge of Intraoperative Floppy Iris Syndrome. Urology 2016;97:40-5.

23. Leibovici D, Bar-Kana Y, Zadok D, et al. Association between tamsulosin and intraoperative "floppy-iris" syndrome. Isr Med Assoc J 2009;11:45-9.

24. Chatziralli IP, Sergentanis TN. Risk factors for intraoperative floppy iris syndrome: a meta-analysis.
Ophthalmology 2011;118:730-5.

25. Wang M, Wang X, Zhao L, et al. Macroglia-microglia interactions via TSPO signaling regulates microglial activation in the mouse retina. J Neurosci 2014;34:3793-806.

26. Chadha V, Borooah S, Tey A, et al. Floppy iris behaviour during cataract surgery: associations and variations. Br J Ophthalmol 2007;91:40-2.

27. Srinivasan S, Radomski S, Chung J, et al. Intraoperative floppy-iris syndrome during cataract surgery in men using alpha-blockers for benign prostatic hypertrophy. J Cataract Refract Surg 2007;33:1826-7.

28. Kaczmarek IA, Prost ME, Wasyluk J. Clinical risk factors associated with intraoperative floppy iris syndrome: a prospective study. Int Ophthalmol 2019;39:541-9.

29. Wen JQ, Hong SM, Chen YD. Risk factor analysis of IFIS in age related cataract surgery. Chinese Hospital Statistics 2018;25:176-8+82.

30. Chang DF, Braga-Mele R, Mamalis N, et al. ASCRS White Paper: clinical review of intraoperative floppy-iris syndrome. J Cataract Refract Surg 2008;34:2153-62.

31. Popescu RM, Ober C, Sevastre B, et al. Complications of cataract surgery in Wistar rats undergoing treatment with tamsulosin. Exp Ther Med 2019;17:137-46.

32. Prata TS, Palmiero PM, Angelilli A, et al. Iris morphologic changes related to alpha(1)-adrenergic receptor antagonists implications for intraoperative floppy iris syndrome. Ophthalmology 2009;116:877-81.

33. Santaella RM, Destafeno JJ, Stinnett SS, et al. The effect of alpha1-adrenergic receptor antagonist tamsulosin (Flomax) on iris dilator smooth muscle anatomy. Ophthalmology 2010;117:1743-9.

34. Wierzchowski T, Wilczynski M, Palenga-Pydyn D, et al. Intraoperative floppy iris syndrome - epidemiology and clinical assessment. Klin Oczna 2016;118:187-90.

35. Goseki T, Ishikawa H, Ogasawara S, et al. Effects of tamsulosin and silodosin on isolated albino and pigmented rabbit iris dilators: possible mechanism of intraoperative floppy-iris syndrome. J Cataract Refract Surg 2012;38:1643-9.

36. Panagis L, Basile M, Friedman AH, et al. Intraoperative floppy iris syndrome: report of a case and histopathologic analysis. Arch Ophthalmol 2010;128:1437-41.

37. Tzamalis A, Malyugin B, Ziakas N, et al. Angiotensin receptor inhibitors as main predisposing factor for intraoperative floppy iris syndrome in women. J Cataract Refract Surg 2019;45:696-7.

38. Bilgin B, Ilhan D, Cetinkaya A, et al. Intraoperative floppy iris syndrome associated with quetiapine. Eye (Lond) 
2013;27:673.

39. Matsuo M, Sano I, Ikeda Y, et al. Intraoperative floppy-iris syndrome associated with use of antipsychotic drugs. Can J Ophthalmol 2016;51:294-6.

40. Unal M, Yucel I, Tenlik A. Intraoperative floppy-iris syndrome associated with chronic use of chlorpromazine. Eye (Lond) 2007;21:1241-2.

41. Wilson ME, Jr., Trivedi RH, Mistr S. Pediatric intraoperative floppy-iris syndrome. J Cataract Refract Surg 2007;33:1325-7.

42. Tint NL, Dhillon AS, Alexander P. Management of intraoperative iris prolapse: stepwise practical approach. J Cataract Refract Surg 2012;38:1845-52.

43. Esen F, Bulut AE, Toker E. Efficacy and safety of lowconcentration, bisulphite-containing, intracameral epinephrine and topical atropine treatments for the prevention of intraoperative floppy iris syndrome. Cutan Ocul Toxicol 2018;37:286-90.

44. Nuzzi R, Arnoffi P, Tridico F. Best Prophylactic Strategy in Groups at Risk of Intraoperative Floppy Iris Syndrome Development: Comparison Between Atropine Instillation and Adrenaline Intracameral Injection. Open Ophthalmol J 2018;12:34-40.

45. Silverstein SM, Rana VK, Stephens R, et al. Effect of phenylephrine $1.0 \%$-ketorolac $0.3 \%$ injection on tamsulosin-associated intraoperative floppy-iris syndrome. J Cataract Refract Surg 2018;44:1103-8.

46. Moore SP, Goggin M. Intraoperative floppy iris syndrome and microincision cataract surgery. J Cataract Refract Surg

Cite this article as: Yang X, Liu Z, Fan Z, Grzybowski A, Wang N. A narrative review of intraoperative floppy iris syndrome: an update 2020. Ann Transl Med 2020;8(22):1546. doi: 10.21037/atm-20-3214
2010;36:2008.

47. Armarnik S, Mimouni M, Rosen E, et al. Modified corneal incisions in intraoperative floppy iris syndrome (IFIS)prone patients. Graefes Arch Clin Exp Ophthalmol 2016;254:123-7.

48. Blau-Most M, Mimouni M, Geffen N, et al. Modified Corneal Incisions for Cataract Surgery in Patients Treated with Tamsulosin: A Prospective Study. Curr Eye Res 2019;44:381-4.

49. Keklikci U, Isen $\mathrm{K}$, Unlu K, et al. Incidence, clinical findings and management of intraoperative floppy iris syndrome associated with tamsulosin. Acta Ophthalmol 2009;87:306-9.

50. Lockington D, Wang Z, Qi N, et al. Modelling floppy iris syndrome and the impact of pupil size and ring devices on iris displacement. Eye (Lond) 2020. [Epub ahead of print].

51. Chang DF, Osher RH, Wang L, et al. Prospective multicenter evaluation of cataract surgery in patients taking tamsulosin (Flomax). Ophthalmology 2007;114:957-64.

52. Lawrentschuk N, Bylsma GW. Intraoperative 'floppy iris' syndrome and its relationship to tamsulosin: a urologist's guide. BJU Int 2006;97:2-4.

53. Flach AJ. Intraoperative floppy iris syndrome: pathophysiology, prevention, and treatment. Trans Am Ophthalmol Soc 2009;107:234-9.

54. AUA Practice Guidelines Committee. AUA guideline on management of benign prostatic hyperplasia (2003). Chapter 1: Diagnosis and treatment recommendations. J Urol 2003;170:530-47. 